\title{
Snorre WAG Pilot - A Case Study
}

\author{
* Stenmark H., ${ }^{\star \star}$ Andfossen P. 0. \\ * Saga Petroleum a. s., Norway \\ ** Petec a. S., Norway
}

\begin{abstract}
Copyright 1995, Steering Committee of the European IOR - Symposium.
This paper was presented at the 8th. European IOR - Symposium in Vienna, Austria, May 15 - 17, 1995

This paper was selected for presentation by the Steering Committee, following review of information contained in an abstract

submitted by the author(s). The paper, as presented has not been reviewed by the Steering Committee.
\end{abstract}

\begin{abstract}
A WAG injection pilot was initiated in February 1994 in the Snorre Field. This resulted in an immediate increase in oil production, as gas export restrictions no longer limited oil rates. In 1994, the total acceleration of oil production due gas injection was estimated at $1.5 \times 10^{6} \mathrm{Sm}^{3}$.
\end{abstract}

Early gas breakthrough was experienced in one well. There has since been limited production of injected gas in only one of the other producers.

A reasonable match of the early pilot behaviour has been obtained with both miscible and immiscible reservoir modelling assumptions. Although laboratory experiments have indicated that conditions are present for a miscible gas injection process in the Snorre Field, immiscible modelling assumptions appear to give the most accurate reproduction of GOR development in the producers. The simulated long term performance is similar for both approaches. The results indicate a positive effect from continued WAG injection.

Uncertainties associated with the degree of vertical segregation combined with the possibility of gas leaking across to the neighbouring faultblock means that further pilot observation is required to verify the simulated results.

\section{Introduction}

The Snorre Field is located in blocks $34 / 4$ and $34 / 7$ on the oil prolific Tampen Spur, $200 \mathrm{~km}$ north-west of Bergen (Fig. 1). The field was put on stream in August 1992, with Saga Petroleum a.s. as the operator. The field contains oil in the lower Jurassic Statford
Formation and in the Upper Member of the Triassic Lunde Formation. The development has been phased, with phase 1 mainly focusing on the Statfjord Formation. Water injection was chosen as the main recovery mechanism [1].

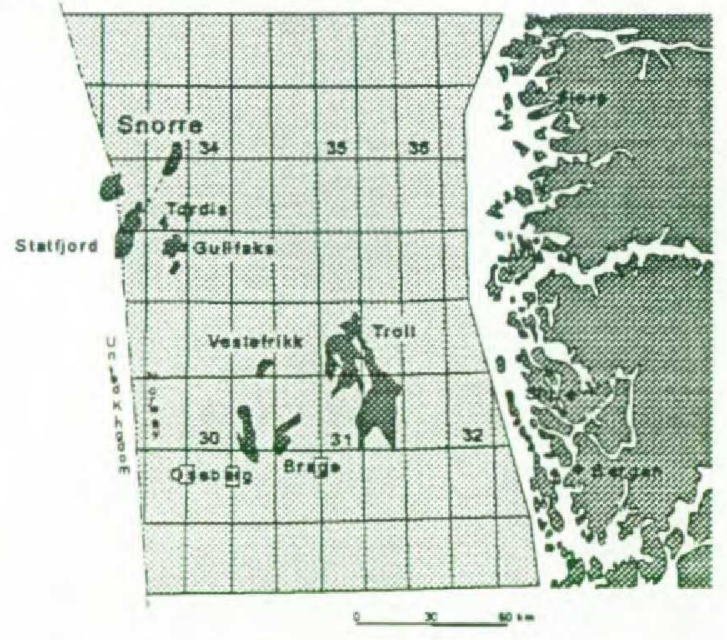

Figure 1. Location of the Snorre Field

Previous reservoir studies [2] showed WAG to be a potentially very attractive oil recovery mechanism for Snorre, significantly increasing oil rates and recovery. Furthermore, WAG was expected to improve Snorre's gas offtake situation, because gas volumes exceeding gas export constraints could be reinjected in the Snorre Field.

Due to the heterogeneous nature of the reservoir, a field pilot test in a limited area of the Statfjord Formation (Fig. 2) was considered necessary. Gas injection commenced in February 1994.

The current plan is to continue WAG injection and evaluation, before a recommendation on future gas disposition strategy will be made later this year. 
The objective of this paper is to:

- summarise the experience from the first year of WAG injection in the Snorre Field

- investigate uncertainties in interpretation of pilot behaviour based on a history matching process using different black-oil simulation modelling approaches

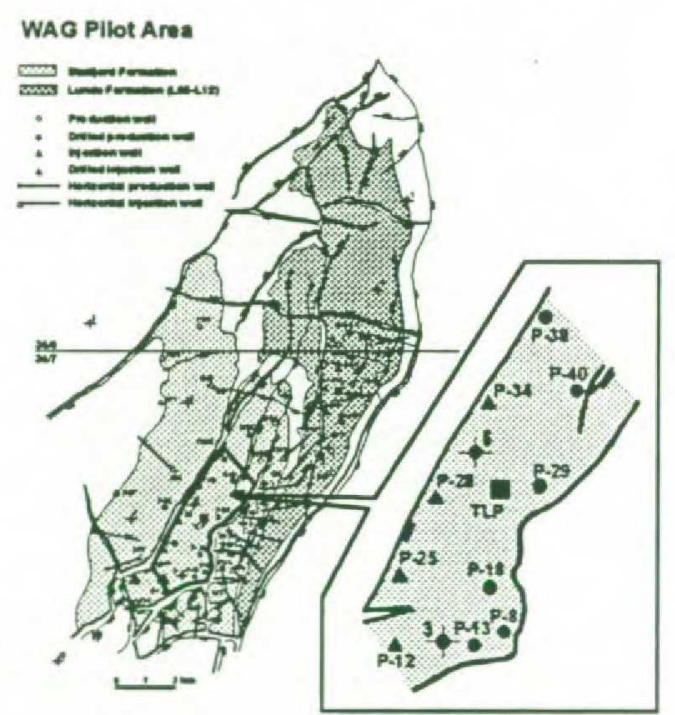

Figure 2. Snorre WAG pilot area

\section{Reservoir description}

The Statfjord Formation consists of fluvially deposited channel belt sandstones, separated by non-reservoir mudstones. The permeabilities are typically 1000 $2000 \mathrm{md}$ in the Upper Statfjord, and 200-1000 md in the Lower Statfjord. It is subdivided into five reservoir zones based on a conceptual allostratigraphic model [3]. These zones are again composed of several allostratigraphical subunits. In zones with high sand content, the sandstones show some tendency to erode into each other, which allows a certain degree of vertical communication. Zones with low sand content have more persistent mudstone layers, creating effective vertical barriers to flow.

\section{Cross-section through WAG pilot area}

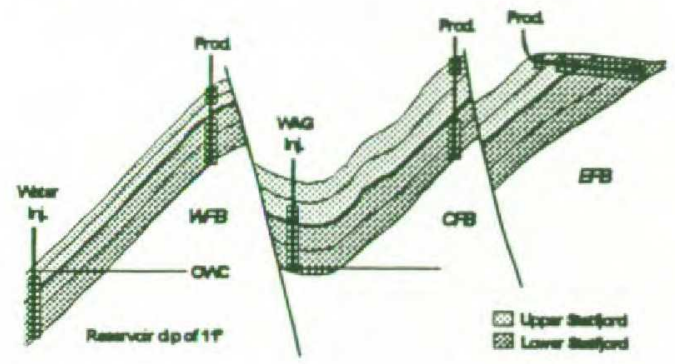

Figure 3. Schematic cross-section of wells in the pilot area

As a consequence of the superior reservoir quality, by far the largest portion of injected gas has entered the Upper Statfjord. Vertical and lateral communication has been proven to be very good by essentially all dynamic data acquired so far. Even so, heterogeneities on a reservoir subzone level are expected to have a major impact on gas flooding performance. A schematic cross-section of the WAG pilot area in the Central Fault Block (CFB) as well as the neighbouring Western and Eastern Faultblocks (WFB, EFB) is shown in Figure 3. Pressure communication exists between CFB and EFB while WFB is separated.

\section{Fluid properties}

The reservoir fluid is a relatively light oil which is highly undersaturated. Reservoir temperature is $90^{\circ} \mathrm{C}$ and the initial reservoir pressure is 383 bar. The saturation pressure varies between 90 and 130 bar and the solution gas/oil ratio (GOR) between 60 and 100 $\mathrm{Sm}^{3} / \mathrm{Sm}^{3}$. The miscibility pressure has been estimated at 283 bar based on slim tube displacement experiments. In order to ensure miscible conditions, the reservoir pressure in the WAG pilot area has been kept above 300 bar during the first year of gas injection.

\section{WAG pilot history}

Screening studies performed in 1991 and 1992 showed that WAG injection in the Snorre Field could potentially be an effective oil recovery method compared to water injection. A WAG pilot test was decided on before committing investments to a large scale WAG implementation. The major factors in favour of WAG injection were summarised as: 
- Increased oil production rates and improved oil recovery due to improved sweep and reduced residual oil saturation

- Improved gas offtake situation, by reinjection of produced gas volumes exceeding the export constraints

The importance of the gas offtake situation has since increased, as oil production is now stable at a level higher than initial design capacity while at the same time the average solution GOR of the produced oil has proved to be higher than what was originally predicted.

Gas injection in the Snorre field commenced in February 1994 in the injector P-25. This resulted in an immediate increase in oil production as gas export restrictions no longer limited oil rates. With the current injection rates and producing GOR, this increase is between 4000 and $8000 \mathrm{Sm}^{3} / \mathrm{SD}$ of oil. In 1994, the total acceleration of oil production due to gas injection was estimated at $1.5 \times 10^{6} \mathrm{Sm}^{3}$.

The injection rate was initially set to about $1.1 \times 10^{6}$ $\mathrm{Sm}^{3} / \mathrm{d}$ to maintain voidage replacement, with a cycle period of three months. After an early gas breakthrough was observed in producer $\mathrm{P}-18$, the injection rate was reduced, corresponding to gas volumes exceeding the contracted export volumes. The average rate has since then been around $0.8 \times 10^{6} \mathrm{Sm}^{3} / \mathrm{d}$ (Fig. 4).

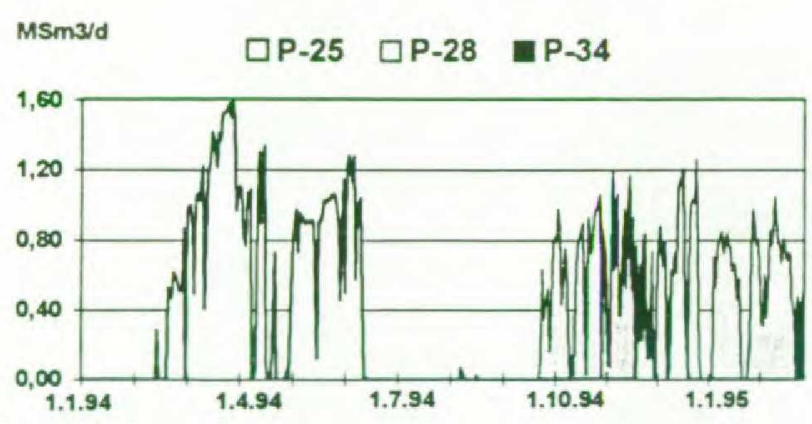

Figure 4: Gas injection history

Gas injection was shut down during the summer of 1994 due to compressor problems. The WAG cycle was reduced to one month per well when gas injection resumed in the autumn, cycling between wells P-25, P28 and P-34 (Fig. 2).

\section{Data Acquisition}

\section{GOR development}

The first gas breakthrough occurred in well P-18 after only one month of gas injection. A sharp increase of GOR to values exceeding $200 \mathrm{Sm}^{3} / \mathrm{Sm}^{3}$ was measured. As oil production was limited by gas processing capacity, the well was shut in. It has since been put on stream for very short periods for data acquisition only.

A gradual GOR increase was observed in the well P-29 about five months after gas injection commenced (Fig. 5). The measured values increased to a maximum of almost $120 \mathrm{Sm}^{3} / \mathrm{Sm}^{3}$ before returning to near initial values.

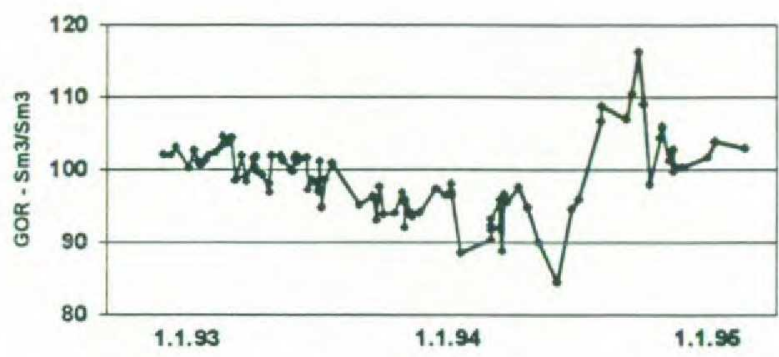

Figure 5. P-29 GOR development

\section{Tracer injection}

In order to monitor the frontal movement of injected gas, tracers have been injected in the gas phase in the wells P-25 (PMCH) and P-28 (PMDH).

When well P-18 was reopened for a few hours of testing one month after breakthrough had occurred, gas tracers from well P-25 was observed. This tracer was introduced to the gas stream about the same time as the breakthrough occurred, indicating that gas continued to move towards and past well P-18 at approximately the same speed even after the well was shut in.

In well P-29, only gas tracers from well P-25 have been detected so far. Although the tested GOR values have returned to near initial values, low concentrations of gas tracers are still present.

\section{P-18 gas monitoring}

A major uncertainty in the interpretation of the WAG results is the rate at which gas accumulates at the top of the structure. Saturation logging of well P-18 was initiated in order to investigate this. 
Temperature-Decay-Time (TDT) logging of the well in September 1994 showed free gas to be present in the uppermost reservoir layer. A repeat TDT run in

Febriary 1995 showed the same interval to be gas filled with a maximum contact movement estimated at $0.5 \mathrm{~m}$. There was no indication of free gas to be present in the intervals below.

These results may be interpreted as a positive indication of only small amounts of gas accumulating updip. An uncertainty associated with the possibility of gas leaking across to the neighbouring fault-block does, however, mean that this can not yet be concluded.

\section{Asphaltenes}

Asphaltenes were found to be a present in the wellbore when well P-18 was re-entered after being shut in for two weeks following the gas breakthrough. Although soluble with chemicals, any potential operational problems caused by this are currently being investigated. Laboratory results have later indicated that asphaltene precipitation will occur at reservoir conditions in the presence of free gas.

\section{Water cut development}

When the WAG pilot was planned, one of the expected sources of information about pilot behaviour was the water-cut development of the producers in the pilot area. Based on simulation models, water production was expected to rise through 1994, and gas injection was predicted to result in significant changes in the development. The actual water production has been lower than predicted, with minor amounts of water being produced from the Lower Statfjord mainiy. The watercut of the wells in the pilot area has been around $5 \%$. A stabilisation and in some instances slight reduction in water-cut development have been observed.

\section{Observation well}

The water injector P-12 in the underlying Lunde L02L05 reservoir is being used as an observation well for the WAG pilot. The well is located slightly to the south of the pilot area, and showed no signs of gas being present in the Statfjord formation when a TDT base log was run early April 1994. Repeat TDT logs will be run at a later stage to monitor any gas front movement in this direction.

\section{Reservoir Simulation}

\section{WAG modelling strategy}

In the reservoir simulation studies, a black oil simulation model (ECLIPSE), which can be run in either immiscible or miscible (solvent) mode has been used.

Previous WAG screening studies have been based on fine-gridded element models covering the WAG pilot area only. After implementation of the WAG pilot, the principal tool for WAG simulation has been the history matched Statfjord simulation model. This has been necessary in order to include effects of activities outside the WAG pilot area.

All simulation cases discussed in this paper are based on a deterministic simulation model covering the Statfjord Formation in the Central and Eastern Fault Block. The grid size in the model is typically 100 by 120 meters areally. The vertical grid definition was initially one layer per reservoir subunit, but was later somewhat refined in the history matching process discussed below.

The parameter maps used in the model are based on digitised net/gross maps with petrophysical parameters contoured from well values.

Laboratory experiments have determined the injection gas to be vaporising multiple contact miscible with the oil at pressures above 283 bar, somewhat below the reservoir operating pressure of 300 to $320 \mathrm{bar}$. The gas-oil mixing and displacement process have been modelled both under miscible and immiscible assumptions.

\section{Miscible vs. immiscible modelling considerations}

Two phase modelling using the ECLIPSE miscible option has been the basis for WAG simulation both in the pre-pilot element model studies and in the historymatching using the Statfjord simulation model.

The key parameter in the mixing rule approach suggested by Todd and Longstaff [4] and used in the miscible mode is the mixing factor, hereafter referred to as the $\omega$-factor. This factor takes into account effects of reservoir heterogeneities and dispersed flow due to incomplete mixing. It will also depend on the gridding of the model. Based on detailed sector modelling, the value used for predictions made with the coarse Statfjord model was set to 0.4 . 
Although laboratory experiments have indicated that conditions are present for a miscible gas injection process in the Snorre Field, immiscible modelling of the gas injection process has also been attempted. The reasons for this are that the immiscible three-phase formulation of Eclipse can model effects not possible to model by the miscible Todd-Longstaff model and that although miscible displacement occurs in the laboratory, some of the oil being exposed to gas in the reservoir will not be miscibly displaced. This will be further discussed below.

While the Todd-Longstaff method provides a simple and convenient way to model miscible gas displacement, certain effects can be modelled more accurately using the immiscible three-phase formulation. The positive effect on oil recovery caused by swelling of a highly undersaturated oil like the Snorre oil, is not modelled explicitly in the ToddLongstaff method, but may implicitly be taken into account by using a higher $\omega$-factor than would otherwise be used for a less undersaturated oil. Also, gas relative permeability hysteresis to express a residual gas saturation higher than the critical gas saturation can not be modelled. This effect may be of importance in simulation of a WAG process, where the residual gas saturation to some extent determines the net amount of gas that can be stored in the reservoir. The three-phase immiscible formulation can handle these effects.

The amount of the oil in contact with gas that will be miscibly displaced compared to the amount of oil being merely swelled, vaporised or otherwise immiscibly displaced is hard to quantify. Immiscible conditions can occur locally under potentially miscible conditions. As an example, consider a case with downdip injection of gas where the viscous/gravitational forces cause the gas to flow in a channel-like manner and where a miscible zone has been established in the leading edge of the gas oil interface. The oil in the gas swept area may then have been miscibly displaced with resulting small residual oil saturations. Behind the leading miscible zone, the channel gas will be compositionally close to the injection gas. Some of this gas will have had minor, if any, compositional exchange with the reservoir oil. If gas injection then stops, the gas channel will have time to spread and mix with the oil. The process of dispersion or spreading of the gas channel will cause insufficient displacement of oil to generate a miscible zone, but will instead mainly cause swelling of the nearby oil. Furthermore, any oil swelled to the extent of been saturated will have lost the potential of becoming miscible with the injection gas.
A useful feature of the three-phase immiscible formulation of Eclipse is the possibility to put a time lag on the rate at which gas is going into solution with the oil. A value between zero and infinite can be entered for the keyword DRSDT, which controls the rate at which the solution gas-oil ratio is allowed to rise. The DRSDT default value is infinite, meaning gas entering a grid cell will instantly go in solution with the oil up to the value of the saturated solution gas-oil ratio. A reduced value of DRSDT may reflect that the contact area between the gas and the oil is limited, and that the amount of oil being instantly exposed to gas is small compared to the total volume of oil present in a grid cell. With time, however, more oil will be exposed.

\section{Miscible results}

The early pilot-behaviour differed from the predictions in that the actual gas breakthrough occurred earlier than predicted and in a different well (Fig 6.). The gas breakthrough was predicted to occur in well P-13 after 3-4 months while it actually occurred in well P-18 after about one month.

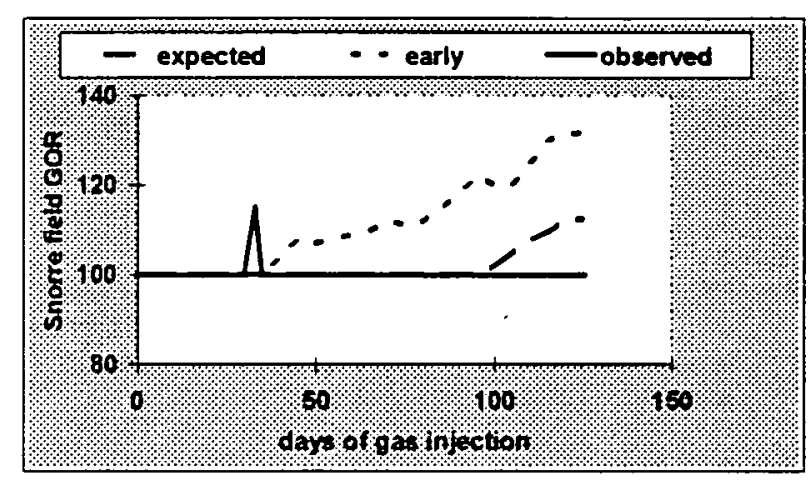

Figure 6. Pre-pilot model GOR vs. observed

Also, the GOR increase following the breakthrough was sharper than for any of the prediction cases. The well had to be shut in to maintain field oil production plateau, and GOR development as a function of time has therefore not been available.

A reasonable match of the early gas breakthrough time was obtained by the following changes in modelling parameters:

- Introduction of transmissibility barriers along the fault north of well P-13

- Introduction of thin grid layers (1/8th of total thickness) in the top of two layers in the Upper Statfjord 
- Increased vertical communication by allowing flow across shale layers thinner than 3 metres

The gas front was diverted away from well P-13 towards well $\mathrm{P}-18$ by introducing transmissibility barriers along a west-easterly fault previously thought to be open. This diversion also reduced the gas breakthrough time significantly.

The refinement of the two layers increased the speed of the gas front by promoting gas override in the top of these layers. A similar short term effect could also have been achieved by lowering the $\omega$-factor. The grid refinement gives a more accurate modelling of gravity dominated gas frontal advancement while any $\omega$-factor reduction in general reduces the effectiveness of the miscible gas displacement process.

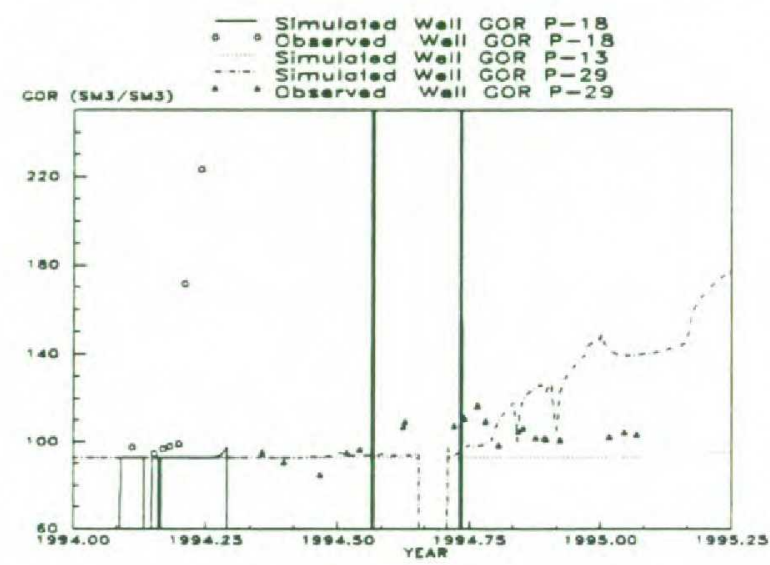

Figure 7. Well GOR with $\omega$-factor $=0.4$

A reasonable match of the observed GOR development was achieved using an $\omega$-factor of 0.4 (Fig. 7). The calculated gas breakthrough occurs about two weeks too late and the subsequent GOR increase is not as sharp as observed. The calculated GOR of well P-29 continues to increase after breakthrough, while the observed GOR stabilises and even decreases. The gas breakthrough in well P-13 is held back by reducing the Statfjord communication across the fault south of well P-18 as discussed earlier.

The comparative case using an $\omega$-factor of 0.0 gave an improved match of the well P-18 behaviour, but appeared somewhat pessimistic in terms of well P-29 GOR development (Fig. 8).

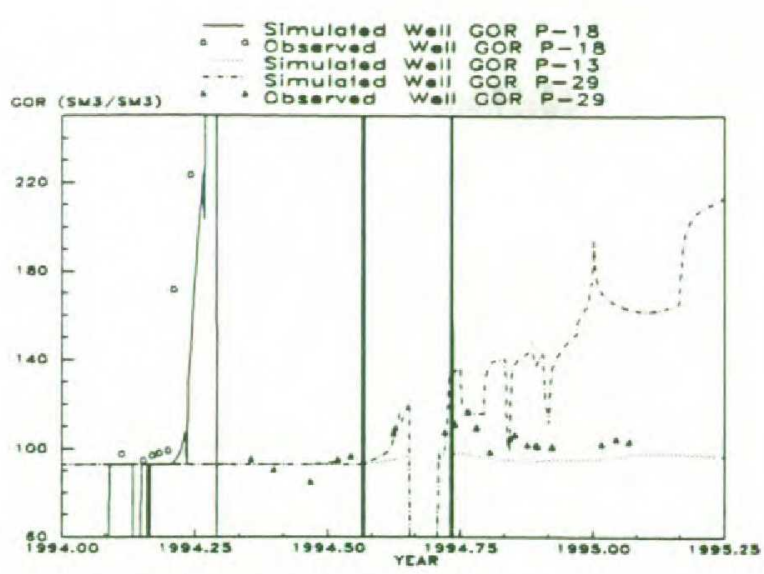

Figure 8. Well GOR with $\omega$-factor $=0.0$

The long term results of a less effective gas displacement process have been modelled as a downside sensitivity case using an $\omega$-factor of 0.0 . Oil and gas production profiles and gas-oil ratios using $\omega$-factors of 0.4 and 0.0 are shown in Figures 9 and 10.

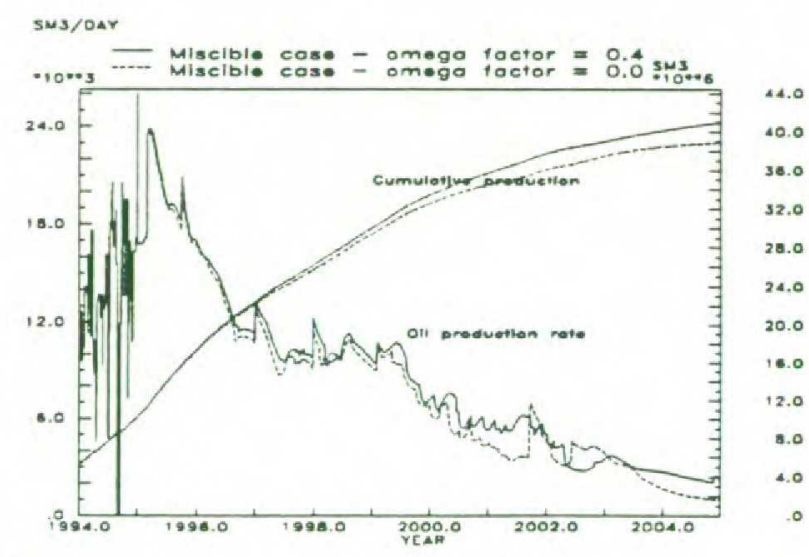

Figure 9. $\omega$-factor sensitivity - oil production

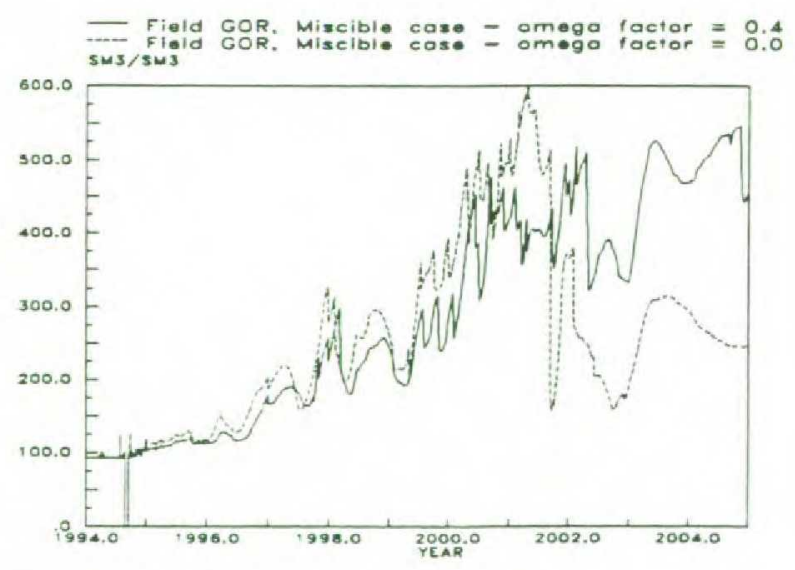

Figure 10. w-factor sensitivity - GOR

WAG injection oil recoveries of the history matched simulation model are similar to the those of the previous prediction model. The WAG injection experience so far therefore supports the expected 
positive effect of WAG injection from the pre-pilot study [2].

\section{Immiscible results}

A match of the WAG pilot behaviour was achieved using a DRSDT value of 0.1 , along with adjustments to the gas relative permeability. These adjustments consisted of the inclusion of hysteresis using a residual gas saturation of 22.1 percent [2] and the use of straight line relative permeability curves. Straight line curves reflect that the gas flow is assumed strongly segregated.

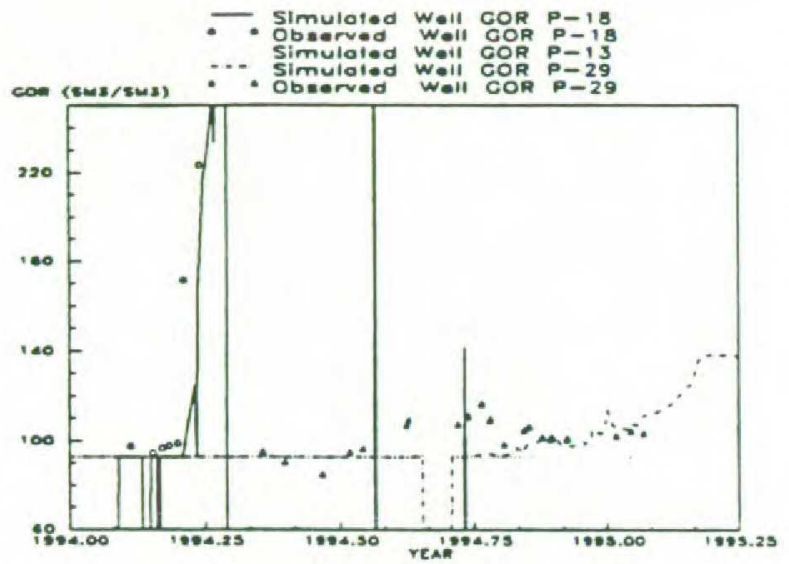

Figure 11. Well GOR for immiscible case

Figure 11 shows the GOR development of the three producing wells expected to be affected by gas. The early gas breakthrough and the subsequent sharp rise in the GOR is well matched. The GOR trend of well P-29 is also modelled well with some period of declining and steady GOR after the initial breakthrough.

The lowered value of the DRSDT parameter helps promoting the initial gas breakthrough in well P-18, but also results in higher GOR's in all wells after gas breakthrough. The use of a residual gas saturation higher than the critical gas saturation has no effect on the initial well P-18 gas breakthrough time and GOR development, but helps in delaying gas breakthrough in the other wells and reduces the GOR in subsequent injection cycles. The straight line relative permeability also helps in promoting the early gas breakthrough as well as the sharp GOR increase in well P-18.

Gas saturation profiles as of 1. January, 1995 are shown on Figures 12 - 15. Figure 12 shows the gas saturations of the top layer, while Figures 13-15 show vertical cross-sections as indicated on Figure 12.

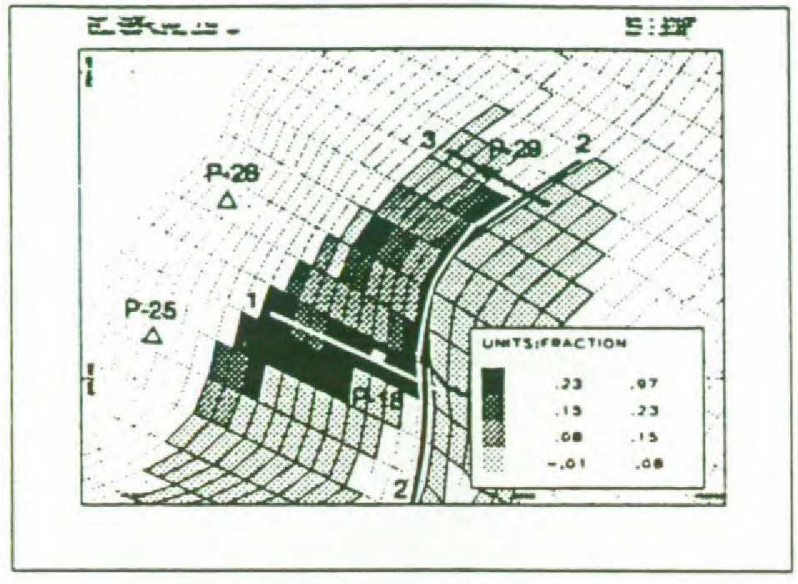

Figure 12. Gas saturation for top layer

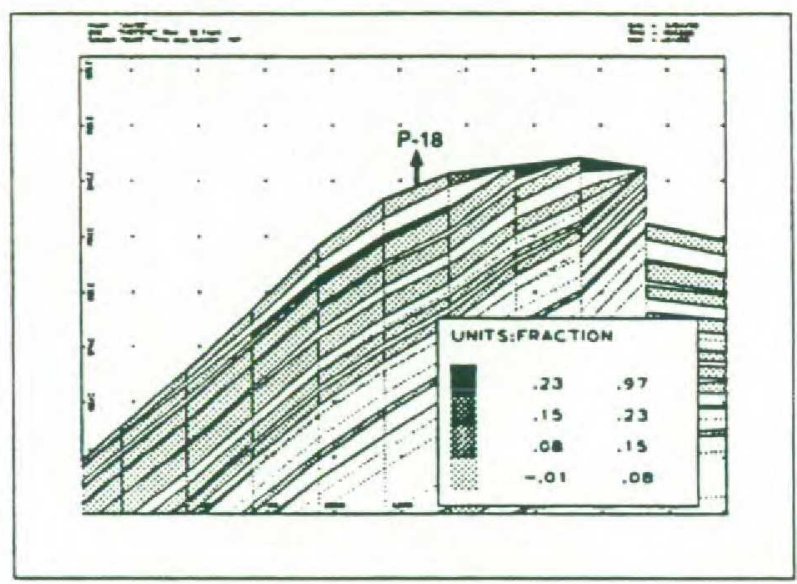

Figure 13. Gas saturation P-18 x-z cross-section 1

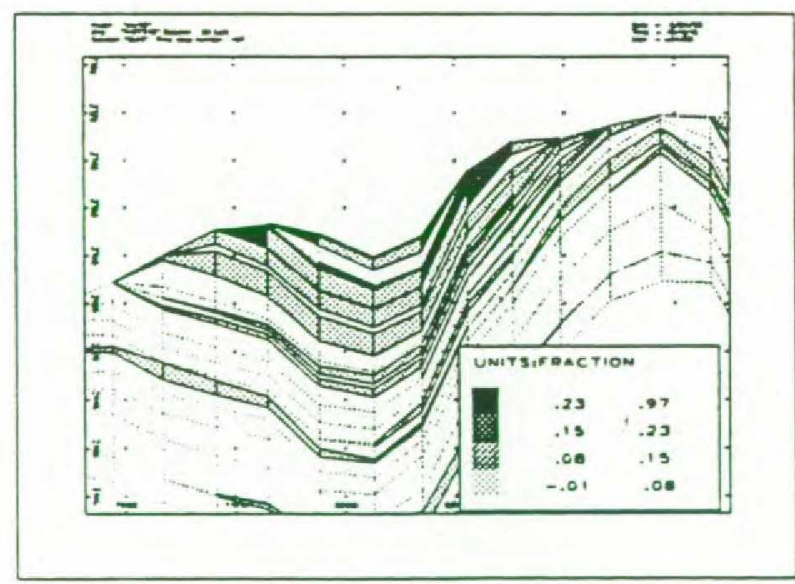

Figure 14. Gas saturation, cross-section no. 2 along EFB boundary 


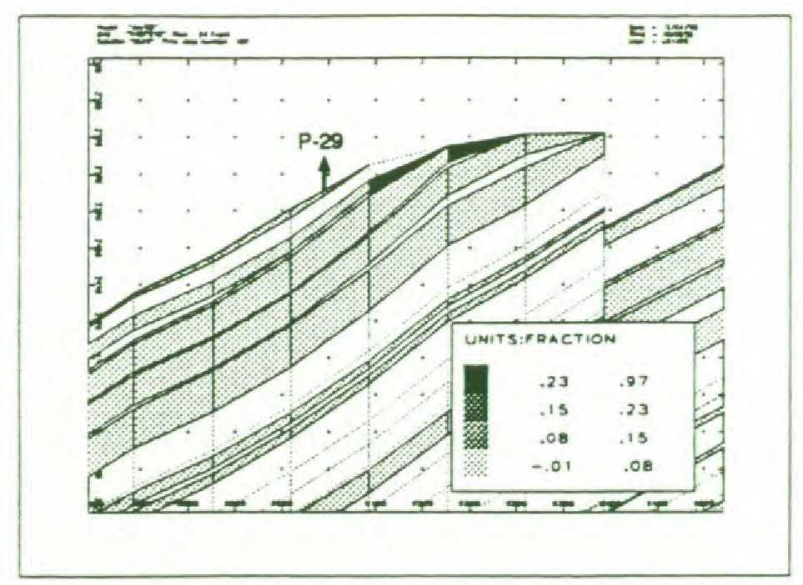

Figure 15. Gas saturation, cross-section no. 3

These simulation results indicate that while some gas flows updip behind the producers, there is also gas between the injectors and the producers. The amount of gas remaining in the flooded areas between the injectors and producers compared to the amount flowing updip behind the producers will to some extent determine the long term success of the WAG injection project. A case where gas primarily fills up the volumes behind the producers may appear effective up to the point where the volumes are filled up, but from then on there will be little further benefit in injecting gas. The simulation results therefore appears promising with respect to the success of the WAG injection process. The main uncertainty is related to any possible communication with the Eastern Fault Block, as was discussed earlier in connection with the well P-18 TDT results.

The long term effects of using different values on the DRSDT are shown in Figures 16 and 17. Lowering the DRSDT has long term negative effects seen as a lower oil production and a higher GOR.

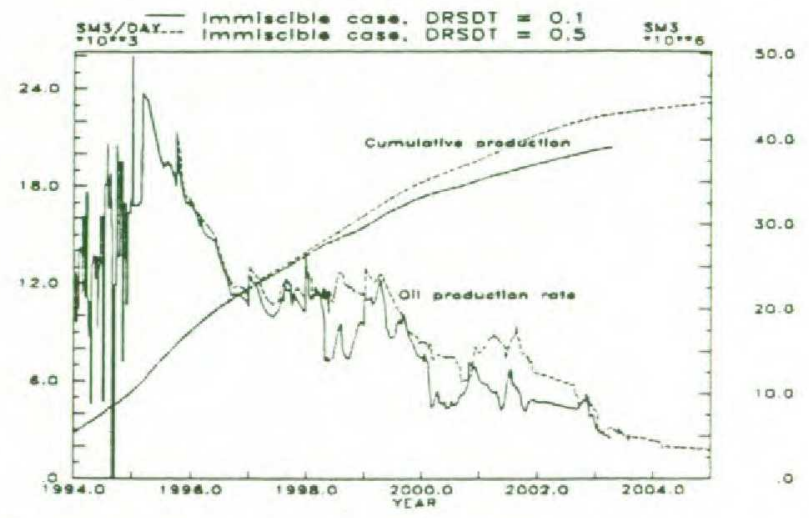

Figure 16. Immiscible DRSDT sensitivity - oil production

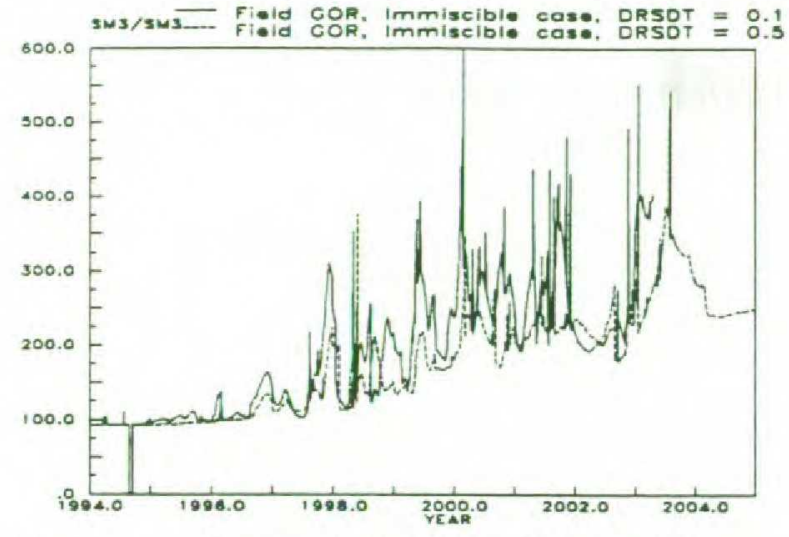

Figure 17. Immiscible DRSDT sensitivity - GOR

The introduction of hysteresis on the gas relative permeability actually has a slight negative long term effect on oil production in the Pilot area (Figs. 18 and 19). The GOR development, however, is lower for the hysteresis case. This may in fact cause an overall positive effect for the Snorre Field since the oil production is limited by gas production constraints.

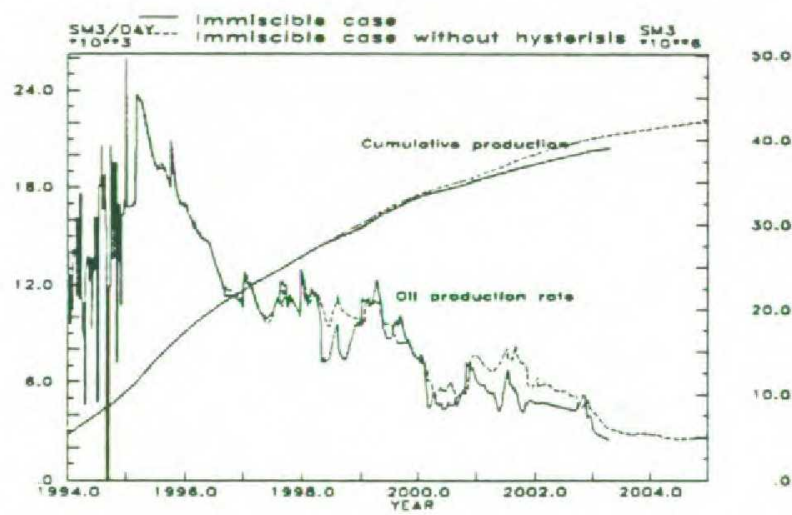

Figure 18. Immiscible hysteresis sensitivity - oil production

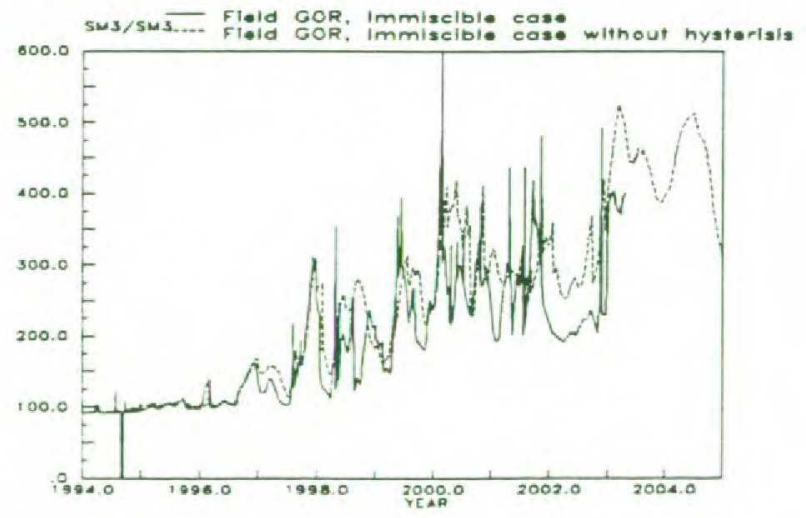

Figure 19. Immiscible hysteresis sensitivity - GOR

The long term differences between the miscible ToddLongstaff base case using an $\omega$-factor of 0.4 and the three-phase immiscible history matched case are shown in Figures 20 and 21. The two cases gave similar results in terms of oil production. The gas-oil 
ratio is generally lower for the immiscible case. This may be caused by the use of hysteresis on the gas relative permeability for the immiscible case, thus retaining more gas in the reservoir.

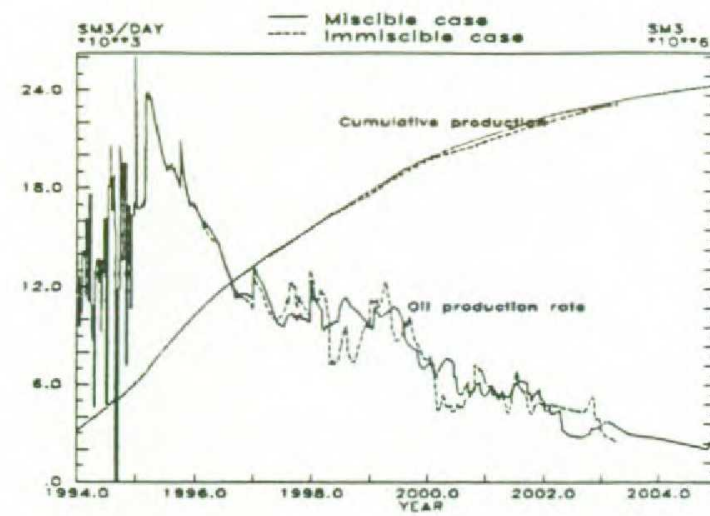

Figure 20. Immiscible vs. miscible - oil production

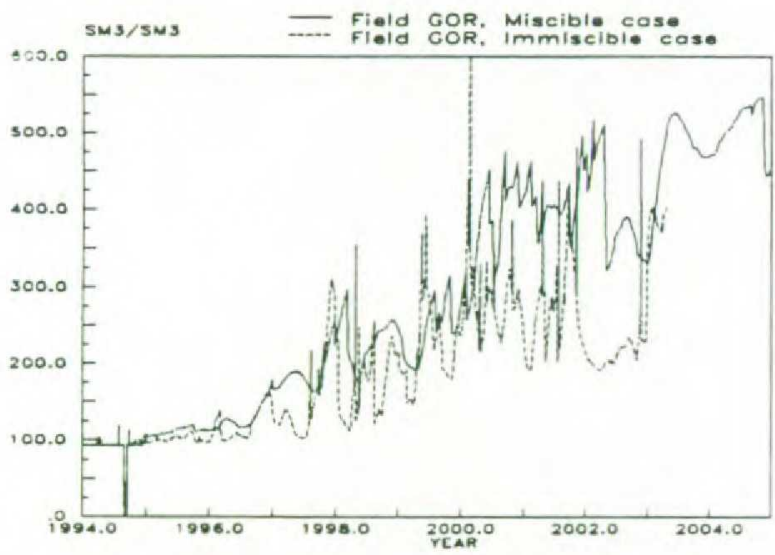

Figure 21. Immiscible vs. miscible - GOR

\section{Conclusions}

- Production experience from the first year of WAG injection in the Snorre Field has shown:

- early gas breakthrough in one well

- late or no GOR development in the other producers

- a small reduction in water cut development

- Accelerated oil production due to gas injection has in this period been in the excess of $1.5 \times 10^{6} \mathrm{Sm}^{3}$ due to gas export constraints no longer being a limitation

- A reasonable history match of the early pilot behaviour has been obtained with both miscible and immiscible modelling assumptions
- Immiscible modelling assumptions appear to give the most accurate reproduction of GOR development in the producers to date

- Long term predictions with history matched models indicate a positive effect from continued WAG injection

- Uncertainties associated with the degree of vertical segregation combined with the possibility of gas leaking across to the neighbouring faultblock mean that further pilot observation is required to verify the simulated results

\section{Acknowledgements}

The authors would like to thank Saga Petroleum and the licence partners on the Snorre Field for the opportunity to work with this project as well as for being allowed to publish the data in this paper.

In addition to the authors, many persons within the Saga organisation have contributed to the work.

The viewpoints expressed in this paper are not necessarily in agreement with those of all the Snorre partners.

\section{References}

1 Nybråten, G., Skolem, E., and Østby, K: "Reservoir simulation of the Snorre Field." Proceedings form the 2nd International Conference on North Sea Oil and Gas Reservoirs, May 8-11, 1989, The Norwegian Institute of Technology, Trondheim, Norway

2 Nybråten, G., Svorstol, I. and Andfossen, P.O.:

"WAG Pilot Evaluations for the Snorre Field." Proceedings from the 7'th European IOR Symposium in Moscow, Russia, October 27-29, 1993.

3 North American Commision on Stratigraphic Nomenclature 1983:

"North American Stratigraphic Code", AAPG Bull. 67, pp 841-875

4 Todd, M. and Longstaff, W.

"The Development, Testing and Application of a Numerical Simulator for Predicting Miscible Flood Performance."

SPE 3484, Feb. 1972. 